\title{
Build-up Systems for calibration tasks
}

\author{
Thomas Kleckers, \\ Hottinger Baldwin Messtechnik GmbH, Im Tiefen See 45, 64293 Darmstadt, Germany, \\ Thomas.Kleckers@HBM.com
}

\begin{abstract}
Build- up systems are force transducers consisting three or more individual sensors working in a parallel arangement. The capacity of the build - up is given as the sum of the capacity of the individual force transducers. The smaller individual force transducers can be calibrated with a relative low effort so that it should be possible to calculate the sensitivity of the complete Build - up system on the base of the individual calibrations in the future.
\end{abstract}

Key words: Force transducers, Build - up -System, Reference load cell, Calibration

\section{Application of Build - up systems}

Upcoming industries such as windmill industry or ship industry are demanding more and more force measurement in the Mega Newton range. As a consequence the demand for calibration of load cells in such ranges is also increasing. Calibration means to refer to an international standard such as the international reference for the mass - the kilogram which can be found in the "Bureau International des Poids et Mesures" in Paris.

Calibration machines of highest precision are consisting masses that known very well and which are related finally to the kilogram in Paris. Force sensors which are subject of calibration are loaded with those masses so that the force is known precisely. The output signal is measured precisely so that the behavior of the sensor is known well. High end machines do reach measurement uncertainties of $0,008 \%$.

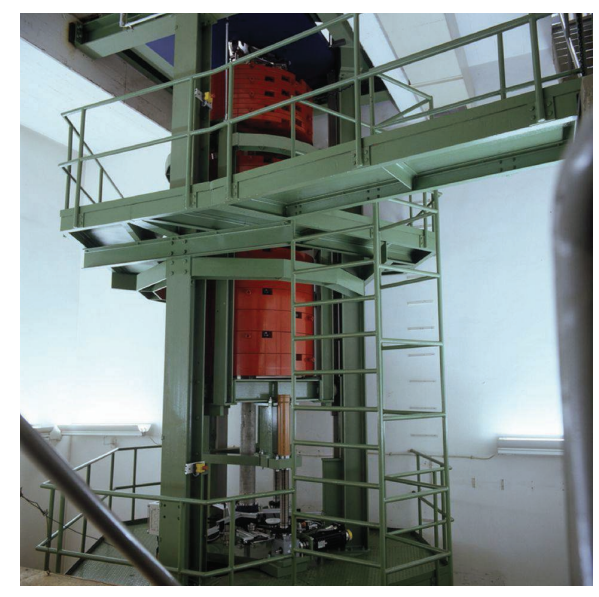

Fig. 1. Masses of a $240 \mathrm{kN}$ Calibration machine with an accuracy of $0.008 \%$.
The concept of masses is limited to relatively low forces. Highest capacity of all calibration machines working with masses - so called dead load machines - in Germany is placed at the PTB in Braunschweig and has a capacity of 2 $\mathrm{MN}$.

With the calibration of higher forces the practical problems of dealing with huge masses are becoming more and more important. Therefore reference machines using hydraulic cylinders. With this concept, the load cell to be calibrated is loaded with a force generated by the hydraulic system. A reference sensor measures the force in serial setup as a master load cell.

For sure the reference force transducer must be more precise that the load cell to be calibrated.

But also the connection of the calibration machines to the international system is done by reference sensors. Therefore reference load cells are used in the machine with closer contact to the international standard. The result of this measurement is compared with the measurement taken in the machine which is subject for the reference measurement.

In case of calibration facilities for forces in the Mega -Newton range the problems are similar to the use of reference sensors in hydraulic machines.

For sure also the reference load cell needs to be calibrated precisely.

The fundamental idea of a build-up system is to calibrate sensors with a precise machine - in best case with a dead load machine- and do the 
parallel setup afterwards. With this concept higher forces can be referred to well-known calibration machines with lower capacity.

The build - up are consisting three or more force transducers with a lower capacity arranged in a mechanical parallel way so that the capacity of the whole system is the sum of all individual sensors. Figure 2 shows the arrangement of such a sensor.

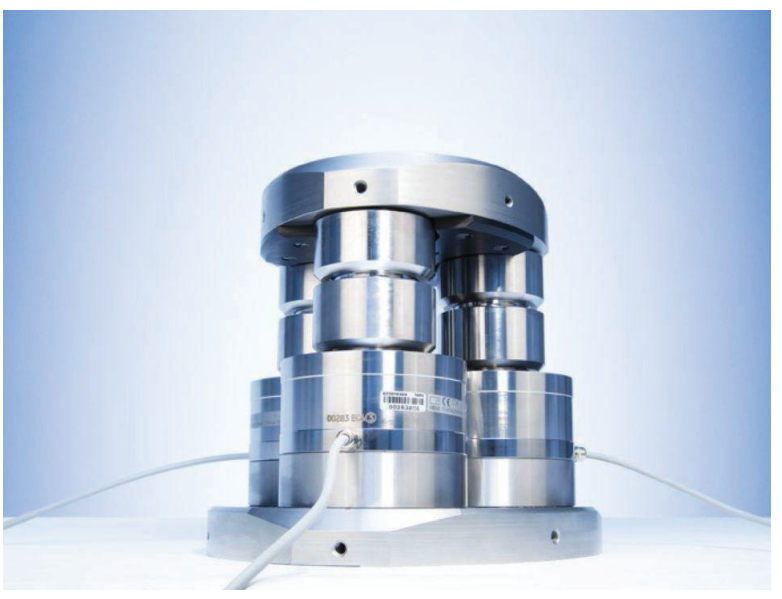

Fig. 2. 3MN Build up System consisting of three 1 $M N$ reference sensors.

\section{Mechanical setup}

Beside the three force sensors a Build-up system requires two massive plates that lead the force to be measured to the reference sensors. Those plates must be massive as the deformation under load should be as low as possible.

Anyhow under higher forces we need to take into account that a little deformation occurs in every part of the system so that the individual force sensors are no more in parallel to the vertical axis of the force transducers. For this reason a trust piece and a pendulum mount are used.

Figure 3 shows the mechanical setup of a high end Build-up: Three sensors with an individual capacity of $3 \mathrm{MN}$ are placed on a massive base plate. The pendulum mount (marked with yellow colour) and the trust piece (marked with green colour) are able to compensate changes in diameter of the base plate as well as of the top plate under load (Poisson effect).

It is very important that all components do have exactly the same height. Therefor a polishing process of the trust pieces as well as of the base plates at the end of the assembly takes place in the pre -mounted system. This guarantees that both load introduction plates are parallel to each other so that the force is introduced with the lowest possible bending moment.
There is always a deflection if strain gauge based force transducers or the required load introduction parts are loaded. The value of defection is depending on the stiffness of the construction. The tolerance of stiffness of the three load cells of importance to keep bending moments out of the system under load. This cannot work in perfection but the design is done in a way so that this is minimized.

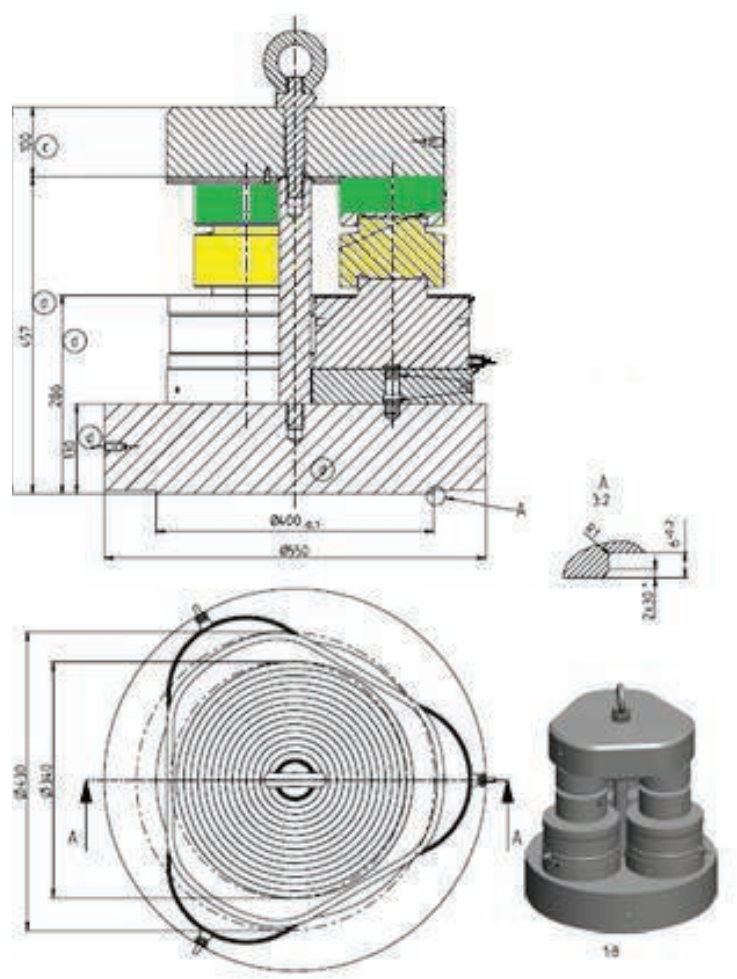

Fig. 3. $6 \mathrm{MN}$ Build up system with all components

\section{Electrical connection}

Strain Gauges sensors are suitable for parallel connection.

To do a parallel connection of load cells in general it is important that the rated output of the force transducers is the same for all sensors connected in one circuit. Furthermore the output resistance is of importance.

In [1] it is shown that the relation $Q$ between rated output and sensitivity needs to be equal for all load cells:

$Q=\frac{R_{o}}{C}$

Where

$\mathrm{R}_{\mathrm{o}} \quad$ Output resistance of the load cell

C Rated output of the load cell 
The output of the load cell is connected to an amplifier system with a very high input resistance so that no current flows. Resistances can be connected in serial according figure 4 .

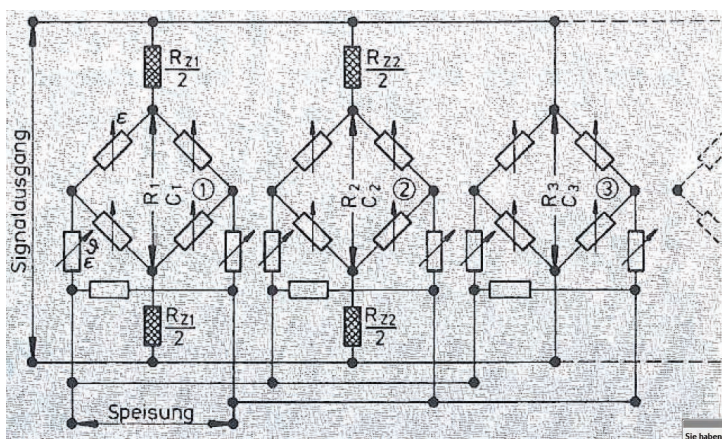

Fig. 4. Parallel setup of three Wheatstone bridges

The dimensions of the resistances have to be calculated in such a way so that $Q$ is equal for all load cells.

\section{Results}

As an example a build-up system with $6 \mathrm{MN}$ was tested.

The three individual sensors as well as the complete system were subject for a calibration in accordance with the ISO376 standard. As a result, the individual sensors as well as the complete systems

Tab. 1: Results of the calibration of the individual sensors

\begin{tabular}{|c|c|c|c|}
\hline Force [kN] & $\begin{array}{c}\text { Output Transducer 1 } \\
{[\mathrm{mV} / \mathrm{V}]}\end{array}$ & $\begin{array}{c}\text { Output Transducer 2 } \\
{[\mathrm{mV} / \mathrm{V}]}\end{array}$ & $\begin{array}{c}\text { Output Transducer 3 } \\
{[\mathrm{mV} / \mathrm{V}]}\end{array}$ \\
\hline 200 & 0,263759 & 0,263733 & 0,263963 \\
\hline 400 & 0,527678 & 0,527552 & 0,527965 \\
\hline 600 & 0,791691 & 0,791474 & 0,792066 \\
\hline 800 & 1,055725 & 1,055414 & 1,05621 \\
\hline 1000 & 1,319869 & 1,319468 & 1,320363 \\
\hline 1200 & 1,584015 & 1,583559 & 1,584596 \\
\hline 1600 & 2,112325 & 2,111751 & 2,112939 \\
\hline 2000 & 2,640616 & 2,639936 & 2,641185 \\
\hline
\end{tabular}

Table 1 shows the results of the calibration of the individual force transducers. It can be seen that the difference in output under a specific load is very low- a result of the individual adjustment of the rated output and the output resistance.

In a next step the sensors are connected in parallel and a calibration of the complete system was performed. Theoretically the capacity of the system is $6 \mathrm{MN}$ but the calibration machine is limited to a capacity of $5 \mathrm{MN}$.

The results are shown in Table 2.

Tab. 2: Results of the calibration of the complete system in comparison with the theoretically calculated rated outputs.

\begin{tabular}{|c|c|c|r|}
\hline Force [kN] & $\begin{array}{c}\text { Output of the of the } \\
\text { system [mV/V] }\end{array}$ & $\begin{array}{c}\text { Theoretical Output } \\
\text { of the system } \\
{[\mathrm{mV} / \mathrm{V}]}\end{array}$ & Difference \\
\hline 1000 & 0,439686 & 0,439966667 & $0,0638 \%$ \\
\hline 2000 & 0,879612 & 0,880193 & $0,0660 \%$ \\
\hline
\end{tabular}

The difference between theoretical output and real measurement shows a difference of $0.06 \%$.

This can be explained by mainly three reasons:

- The adjustment of rated output and output resistance is afflicted with a tolerance

- The stiffness is also afflicted with a certain tolerance. The result that the force is not exactly divided on the three sensors. As a result of this linearity errors of the individual force transducers become of importance.

- The uncertainty of the calibration machine is also of importance. The sensors were tested on a machine wiith an accuracy of $0.02 \%$

For sure it is possible to use three individual amplifier systems to overcome the first point.

\section{Conclusion}

Build-up systems will help to increase the possibility for calibrations in the Mega-Newton range in the future. Current designs do reach the accuracy class of 00 according the international ISO376 standard.

First results show that it should be possible to transfer the accuracy of a lower force range to the Mega-Newton range by using this technology.

\section{References}

[1] Jürgen Paetow, Parallelschaltung von DMS Wägen und Dosieren Ausgabe 6 / 1987 